\title{
Benazepril affects integrin-linked kinase and smooth muscle a-actin expression in diabetic rat glomerulus and cultured mesangial cells
}

Honglin Niu ${ }^{1,2+}$, Lei $\mathrm{Nie}^{3,4+}$, Maodong Liu ${ }^{1,2}$, Yanqing Chi ${ }^{1,2}$, Tao Zhang ${ }^{1,2}$ and Ying $\mathrm{Li}^{1,2^{*}}$

\begin{abstract}
Background: Diabetic nephropathy (DN) is the leading cause of chronic kidney disease and is associated with excessive cardiovascular morbidity and mortality. The angiotensin converting enzyme inhibitor (ACEI) benazepril has been shown to slow the progression of chronic renal disease and have beneficial effects in patients with a combination of chronic renal disease and cardiovascular disease. Transforming growth factor- $\beta_{1}$ (TGF- $\beta_{1}$ ) plays a central role in the pathogenesis and progression of DN. Integrin-linked kinase (ILK) can modulate TGF- $\beta_{1}$-induced glomerular mesangial cell (GMC) injury, which is a prominent characteristic of renal pathology in kidney diseases. As an integrin cytoplasmic-binding protein, ILK regulates fibronectin (FN) matrix deposition and the actin cytoskeleton. Smooth muscle a-actin ( $a-S M A)$ is involved in progressive renal dysfunction in both human and experimental renal disease.
\end{abstract}

Methods: To explore the mechanisms of benazepril's reno-protective effects, we examined the expression of TGF- $\beta_{1}$, ILK, and $a-S M A$ in GMC exposed to high glucose (HG) and in the kidneys of streptozotocin (STZ)-induced diabetic rats using real-time quantitative RT-PCR and western blot analysis. To elucidate the mechanism(s) of the effect of benazepril on GMC cellular processes, we assessed the effect of benazepril on Angiotensin II (Ang II) signalling pathways using western blot analysis.

Results: The expression of TGF- $\beta_{1}$, ILK, and a-SMA increased significantly in the diabetic group compared with the control group. Benazepril treatment inhibited the expression of these genes in DN but failed to rescue the same levels in the control group. Similar results were found in GMC treated with HG or benazepril. Ang II increased ERK and Akt phosphorylation in the HG group, and benazepril could not completely block these responses, suggesting that other molecules might be involved in the progression of DN. Our findings suggest that benazepril decreases ILK and a-SMA expression, at least in part, by affecting the interactions between Ang II and TGF- $\beta_{1}$.

Conclusions: The findings described here support the hypothesis that the HG milieu of diabetes increases TGF- $\beta_{1}$ secretion, which increases the synthesis of ILK and a-SMA that are involved in the progression of DN. This might be an important mechanism of the benazepril renal-protective function in the pathogenesis of DN.

Keywords: Diabetic nephropathy, Integrin-linked kinase, Smooth muscle a-actin, Benazepril, Glomerular mesangial cells

\footnotetext{
* Correspondence: liyinghebei@126.com

${ }^{\dagger}$ Equal contributors

'Department of Nephrology, The Third Hospital of Hebei Medical University,

Shijiazhuang 050051, China

${ }^{2}$ Key Laboratory of Kidney Diseases of Hebei Province, Shijiazhuang 050071,

China

Full list of author information is available at the end of the article
}

C Biomed Central
(C) 2014 Niu et al.; licensee BioMed Central Ltd. This is an Open Access article distributed under the terms of the Creative Commons Attribution License (http://creativecommons.org/licenses/by/4.0), which permits unrestricted use, distribution, and reproduction in any medium, provided the original work is properly credited. The Creative Commons Public Domain Dedication waiver (http://creativecommons.org/publicdomain/zero/1.0/) applies to the data made available in this article, unless otherwise stated. 


\section{Background}

Diabetic nephropathy (DN) is the leading cause of chronic kidney disease worldwide and contributes to significant morbidity and mortality of diabetic patients. Approximately one-third of diabetic patients have progressive deterioration of renal function and ultimately require dialysis or transplantation [1]. This number is expected to rise dramatically as a result of the growing incidence of diabetes and the aging population [2,3]. The pathophysiological mechanisms of DN are incompletely understood, but numerous factors contribute to the pathogenesis and progression of DN. Transforming growth factor- $\beta_{1}$ (TGF$\beta_{1}$ ) can induce the accumulation of extracellular matrix (ECM) components, including collagens, fibronectin (FN) and laminin in the glomeruli and the interstitium of the kidney. TGF- $\beta_{1}$ expression regulates PINCH-1-integrinlinked kinase (ILK)-alpha-parvin complex formation and contributes to glomerular mesangial cell (GMC) proliferation and hypertrophy [4]. ILK, a cytoplasmic-binding serine/threonine protein kinase, is physically connected to the actin cytoskeleton and actin-binding protein $\mathrm{CH}$ ILKBP, which is an important step in the development and progression of glomerular failure observed in several kidney diseases [5]. The GMCs, which have characteristics of a modified smooth muscle cell, occupy the central position in the renal glomerulus. The marker gene smooth muscle $\alpha$-actin ( $\alpha$-SMA) was detected within the juxtamedullary glomeruli during foetal life. When glomerular development is completed after postnatal day 10, $\alpha$-SMA expression is no longer present in the glomerulus [6]. GMCs can be activated by local injury, and activated cells are major sources of ECM synthesis, which affect the progression of renal dysfunction in human and experimental renal diseases [3,7].

Angiotensin II (Ang II) is considered to be involved in the majority of pathological processes that result in DN. Increased Ang II activity causes hypertrophy of GMCs. Ang II promotes the production of TGF- $\beta_{1}$ that leads to progressive renal damage [8-10]. The death incidence due to cardiovascular disease is three times higher in patients with DN than in diabetic patients without signs of renal failure [11]. Because Ang II has an essential role in renal and cardiovascular pathophysiology, angiotensinconverting enzyme inhibitors (ACEI) have been shown to have beneficial effects on renal and cardiovascular diseases [11,12]. Benazepril, an ACEI, provides protection against the progressive deterioration of renal function in patients with renal diseases [13]. In this study, we investigate the effect of benazepril on the renal expression of TGF- $\beta_{1}$, ILK and $\alpha$-SMA in rat DN induced by streptozotocin (STZ) and the effect of benazepril on the expression of these genes associated with Ang II signalling pathway in GMCs. Our work demonstrates the renoprotective effects of benazepril in vivo and in vitro.

\section{Methods}

\section{Animal model and glomerular isolation}

Animal care methods and treatment were conducted in accordance with the guidelines of the Institutional Animal Care and Use Committee of Hebei Medical University and the study protocol was approved by the ethical committee of Hebei Medical University, Shijiazhuang, China (Additional file 1). Male Sprague-Dawley rats weighing 180-250 g were purchased from the Experimental Animal Academy of Chinese Medical Science Institute (Beijing, China). Diabetes was induced by a single tail-vein injection of STZ (Sigma-Aldrich, USA) at a dose of $65 \mathrm{mg} / \mathrm{kg}$ body wt; the STZ was freshly prepared in $0.1 \mathrm{~mol} / \mathrm{L}$ citrate buffer $(\mathrm{pH} 4.5)[14]$. Age-matched male non-diabetic control rats were injected with an equal volume of citrate buffer. Seventy-two hours after the STZ administration, the induction of diabetes was confirmed by measurement of the blood glucose concentration with the OneTouch II blood glucose meter (Johnson \& Johnson, USA). The rats with blood glucose $\geq 16.7 \mathrm{mmol} / \mathrm{L}$ were considered to have diabetes. The rats were randomly divided into 3 groups: normal control group (NC, $n=12)$; diabetic nephropathy group (DN, $n=12$ ); and diabetic nephropathy treated with benazepril (ACEI, $n=12$ ). The diabetic rats were treated with benazepril (Beijing Novartis Pharmacy, China) at $10 \mathrm{mg} / \mathrm{kg}$ per day for 6 weeks by remedial perfusion of the stomach from the third day after the establishment of DN. All of the rats were kept individually in metabolic cages to collect 24-hour urine for the measurement of the 24-hour urinary protein $(\mathrm{TP} / 24)$ at 8 weeks. Blood pressures (BP) were obtained using the Non-Invasive BP system (Kent Scientific Corp, Torrington, CT), and blood samples were collected from the inferior vena cava. The blood glucose (Glu), serum creatinine (Scr), and blood urea nitrogen (BUN) were measured as previously described[15]. The right kidney from each rat was dissected, rinsed with cold saline, placed in the TissueTek O.C.T. compound (Sakura Finetek USA, Torrance, CA), snap frozen in liquid nitrogen and stored at $-80^{\circ} \mathrm{C}$ until further analysis. The renal cortex of the left kidney from each rat was cut into small pieces, and the glomeruli were isolated by the mechanical graded sieving technique. After isolation, the purity of the final suspension was determined by phase contrast microscopy. On average, tubular contamination was less than $5 \%$. The glomerular suspension was used to isolate proteins and RNA [14].

\section{Histological analysis, immunohistochemistry and immunofluorescent staining}

Frozen sections $(5 \mu \mathrm{m})$ of kidney were fixed and stained with haematoxylin and eosin (H \& E) staining and periodic acid schiff (PAS) staining. For the immunohistochemistry 
staining, the frozen sections were fixed in pre-cooled acetone $\left(-20^{\circ} \mathrm{C}\right)$ for $5 \mathrm{~min}$. After being washed 3 times in PBS and treated with $0.3 \% \mathrm{H}_{2} \mathrm{O}_{2}$ for $10 \mathrm{~min}$, the slides were incubated with anti-CD68 (a marker gene for macrophage, \#sc-9139), CD3E (a marker gene for T lymphocyte, \#sc-1127), and TGF- $\beta_{1}$ antibodies (\#sc-146, Santa Cruz Biotechnology, Santa Cruz, CA) overnight at $4^{\circ} \mathrm{C}$. The slides were incubated with a biotinylated secondary antibody, which was followed by the Avidin Biotin Complex $(\mathrm{ABC})$ Method for visualisation ( $R \& D$ Systems, Minneapolis, MN). For glomerular assessment, mesangial area (percentage of glomerular positive stained) was quantitated from 10 glomeruli per kidney per animal using Image J (NIH, Bethesda, MD) [16]. For ILK and $\alpha$-SMA immunofluorescent staining, the frozen sections were permeabilised with $0.1 \%$ Triton X-100 in PBS for 10 minutes and exposed to $1 \times$ SuperBlock Reagent (Thermo scientific, Rockford, lL) with 5\% normal goat serum for 1 hour. The sections were incubated with an anti-ILK antibody (1:200, \#sc-13075, Santa Cruz Biotechnology, Santa Cruz, CA) or an anti- $\alpha$-SMA antibody (1:500, \#A2547, Sigma-Aldrich, St. Louis, MO) and then incubated with a $\mathrm{Cy} 3$ conjugated-goat antirabbit IgG secondary antibody (for ILK, \#A10520) or an Alexa Fluor 488 conjugated goat anti mouse IgG secondary antibody (for $\alpha$-SMA, \#R37120, Life Technologies, Grand Island, NY). Images were acquired by laserscanning confocal microscopy with $20 \times$ objectives (Zeiss LSM 510 meta) after being mounted with the ProLong gold anti-fade reagent with DAPI (Invitrogen, Carlsbad, CA).

\section{GMCs culture}

GMCs were isolated from the glomerulus of four- to sixweek-old Sprague-Dawley rats according to published methods $[17,18]$ and were maintained in Dulbecco's modified Eagle's medium (DMEM, GIBCO, Carlsbad, CA) supplemented with $10 \%$ foetal bovine serum, $5.5 \mathrm{mmol} / \mathrm{L}$ glucose (normal glucose), $100 \mathrm{U} / \mathrm{ml}$ penicillin, $100 \mathrm{mg} / \mathrm{ml}$ streptomycin, $300 \mathrm{mg} / \mathrm{ml} \mathrm{L}$-glutamine at $37^{\circ} \mathrm{C}$ in a $5 \% \mathrm{CO}_{2}$ incubator. To study the expression of TGF- $\beta_{1}$, ILK and $\alpha$-SMA, subconfluent GMCs were cultured in the presence of $5.5 \mathrm{mmol} / \mathrm{L}$ glucose (normal glucose group, NG), $5.5 \mathrm{mmol} / \mathrm{L}$ glucose plus $24.5 \mathrm{mmol} / \mathrm{L}$ mannitol (mannitol group, MG), $30 \mathrm{mmol} / \mathrm{L}$ glucose (high glucose group, HG), or $30 \mathrm{mmol} / \mathrm{L}$ glucose with benazepril $10 \mu \mathrm{mol} / \mathrm{L}$ (high glucose + benazepril group, ACEI) for periods of 3, 6, 12, 24, 48 and $72 \mathrm{~h}$. The Ang II treatment was carried out in the four different types of medium used in the previous experiments for $32 \mathrm{~h}$, and then serum starved with the same medium with the addition of $0.5 \%$ FBS for $16 \mathrm{~h}$, followed by treatment with Ang II for $5 \mathrm{~min}$ (100 nM, Sigma-Aldrich, St. Louis, MO).

\section{RNA isolation and real-time quantitative RT-PCR}

The total RNA was isolated from isolated glomeruli or GMCs with TRIzol (Invitrogen, Carlsbad, CA). The reverse transcriptase reactions were performed using the QuantiTect Reverse Transcription Kit (Qiagen, Valencia, CA). Real-time PCR was performed in triplicate on this cDNA using the ABI Prism 7700 sequence-detection system (Applied Biosystems, Foster City, CA). Designed Taqman $^{\circ}$ probes Mm01178820_m1, Mm01274281_g1 and Mm03944483_s1 for TGF- $\beta_{1}$, ILK, and $\alpha$-SMA were used following the manufacturer's instructions (Applied Biosystems, Foster city, CA). The fold-change analysis was based on the normalised RNA levels by $\beta$-actin in the same sample.

\section{Western blotting}

Tissue samples or cells were lysed in ice-cold RIPA buffer (150 mM NaCl, $50 \mathrm{mM}$ Tris-HCl, pH 8.0, 1.0\% NP-40, $0.5 \%$ sodium deoxycholate, $0.1 \%$ SDS) supplemented with a complete proteinase inhibitor (Roche Applied Sciences, Indianapolis, IN) and phosphatase inhibitor cocktails (Sigma-Aldrich, St. Louis, MO). Equal amounts of protein were resolved by SDS-PAGE and transferred to a PVDF membrane (BioRad, Hercules, CA) and blocked with antiTGF- $\beta_{1}$, ILK (Santa Cruz Biotechnology, Santa Cruz, CA), $\alpha$-SMA (Sigma-Aldrich, St. Louis, MO), phospho-MAPK $\mathrm{p} 44 / 42\left(\mathrm{Thr}^{202} / \mathrm{Tyr}^{204}\right.$, \#9101), total MAPK $\mathrm{p} 44 / 42$ (\#9102), phospho-Akt ( $\left.\mathrm{Ser}^{473}, \# 4060\right)$, and total Akt (\#9272, Cell Signaling Technologies, Danvers, MA), followed by HRP-conjugated anti-rabbit or -mouse secondary antibodies (\#7074 or \#7076, Cell Signaling Technology, Danvers, MA). The blots were developed using an ECL system (PerkinElmer, Boston, MA). GAPDH (\#2118, Cell Signaling Technology, Danvers, MA) was used as the loading control. The films were scanned and quantitative analysis of the ratio of phosphorylated to total MAPK p44/42, Akt or TGF- $\beta_{1}$, ILK, and $\alpha$-SMA from

Table 1 Body weight, blood pressure, serum and urinary tests in different groups

\begin{tabular}{lllllll}
\hline Group & BW $(\mathbf{g})$ & BP $(\mathbf{m m H g})$ & Glu $(\mathbf{m m o l} / \mathbf{L})$ & TP/24 (ml) & Scr $(\boldsymbol{\mu m o l} / \mathbf{L})$ & Bun $(\mathbf{m m o l} / \mathbf{L})$ \\
\hline NC & $227.0 \pm 21.8$ & $105.3 \pm 6.8$ & $6.97 \pm 0.81$ & $6.82 \pm 1.97$ & $48.32 \pm 2.37$ & $7.99 \pm 0.58$ \\
DN & $158.4 \pm 9.3^{*}$ & $142.4 \pm 5.1^{*}$ & $35.63 \pm 2.13^{*}$ & $25.23 \pm 4.23^{*}$ & $72.85 \pm 4.97^{*}$ & $18.32 \pm 1.96^{*}$ \\
ACEl & $187.6 \pm 9.1^{*} \#$ & $123.4 \pm 4.4^{*} \#$ & $21.71 \pm 3.87^{*}$ & $15.12 \pm 2.43^{*} \#$ & $57.34 \pm 3.79^{*} \#$ & $12.02 \pm 1.09^{*} \#$ \\
\hline
\end{tabular}

Values are shown as the mean \pm S.E. ${ }^{*}, P<0.05$ vs. control; $\#, P<0.05$ vs. DN. 


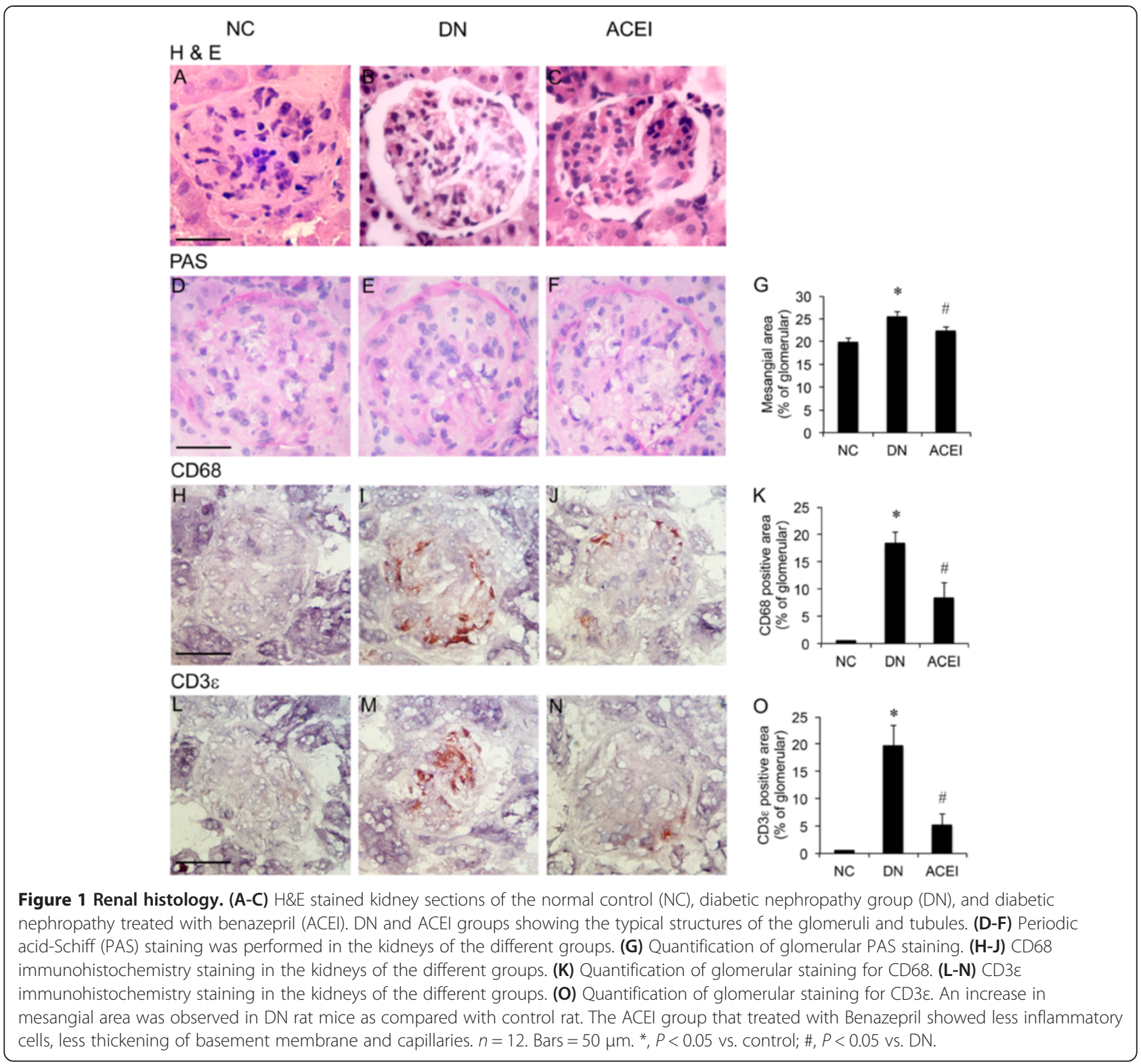

three independent experiments using Kodak 1D 3.5 software (Rochester, NY).

\section{Statistical analysis}

All of the values are expressed as the mean \pm S.E. The significance of the results was assessed by a 2-tailed non-parametric pair $t$-test (Mann-Whitney-U) or a twoway ANOVA with Bonferroni post hoc test (for $>2$ groups). $P<0.05$ was considered statistically significant.

\section{Results}

Body weight, systolic blood pressure and laboratory tests As shown in Table 1, the body weight of DN rats was less than that of the control animals. The benazepril group grew significantly more than the animals with $\mathrm{DN}$ but slightly less than the normal counterparts. The DN rats developed mild hypertension, and a significantly increased BP was observed at 8 weeks in the rats. Benazepril treatment produced a significant decrease in the BP. The blood glucose levels of all of the DN groups were significantly higher than those of the control or benazepril treatment groups $(P<$ $0.05)$. The blood glucose levels in the benazepril treatment group were lower than in the $\mathrm{DN}$ group $(P<0.05)$. As TP/ 24 , Scr and BUN increased significantly in the DN group compared with the control group, Benazepril treatment significantly blunted this decrease $(P<0.05)$.

\section{Renal histology}

We compared the renal histology among the 3 groups. $\mathrm{H}$ \& E staining and PAS staining of the kidney from the 


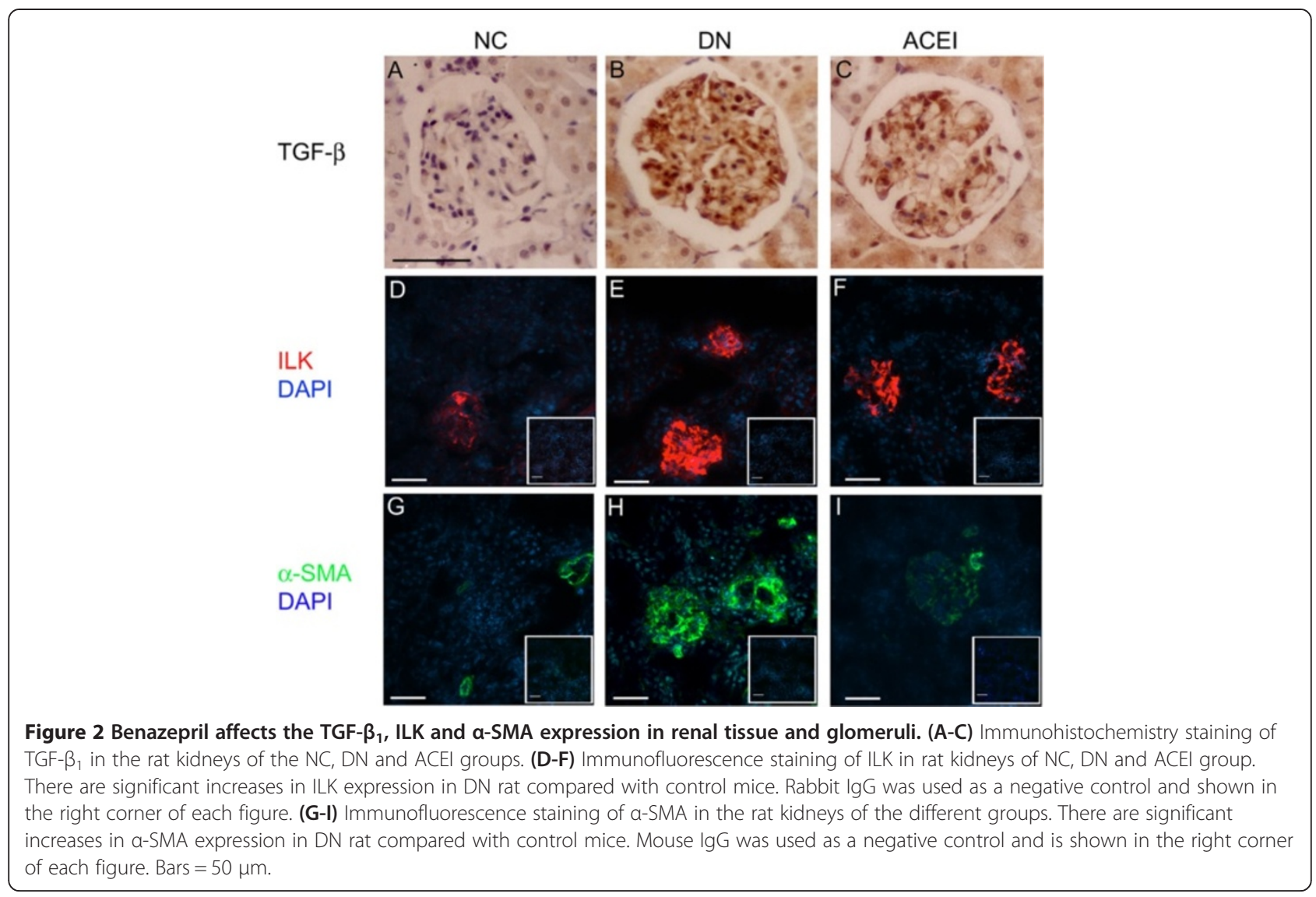

control group revealed a normal glomerulus surrounded by Bowman's capsule and proximal and distal convoluted tubules without any inflammatory changes. The $\mathrm{DN}$ rats showed renal tubule atrophy (Figure 1A-C), thickening of the basement membrane (Figure 1D-G) and degenerated glomeruli infiltrated by inflammatory cells (Figure 1H-O). The ACEI group showed significatly fewer inflammatory cells, reduced thickening of the basement membrane and capillaries compared with DN group (Figure 1A-O).

\section{Benazepril affects TGF- $\beta_{1}$, ILK and $\alpha$-SMA expression in renal tissue}

To further examine the development of the histological changes in diabetes, we examined the molecular pathology that is classically associated with DN. TGF- $\beta_{1}$ expression has emerged as a key participant in the cascade of events that leads to DN [19]. Immunohistochemistry staining showed that the basal level of TGF- $\beta_{1}$ expression was barely detectable in the control rat kidney (Figure 2A). The DN group showed strongly positive TGF- $\beta_{1}$ expression, mainly distributed in the glomerulus, GMCs, and tubular interstitium(Figure 2B). Benazepril treatment significantly decreased the TGF- $\beta_{1}$ expression in the renal tissue (Figure 2C). Immunofluorescence staining for ILK and $\alpha$-SMA expression, paralleled with that for TGF- $\beta_{1}$, was faintly positive (ILK, Figure 2D) or undetectable $(\alpha-$ SMA, Figure 2G) in the rat renal glomerulus and the GMCs in the control group. Strongly positive staining was observed in the rat glomerulus and the GMCs in the DN group (ILK, Figure 2E; $\alpha$-SMA, Figure $2 \mathrm{H}$ ). Benazepril treatment significantly decreased the expression of ILK and $\alpha$-SMA in the rat glomerulus (Figure $2 \mathrm{~F}$ and I), suggesting that the beneficial effects of benazepril were mainly mediated by the inhibition of TGF- $\beta_{1}$, ILK and $\alpha$ SMA expression in diabetic renal tissue.

\section{Benazepril affects TGF- $\beta_{1}$, ILK and $\alpha$-SMA expression in glomeruli}

TGF- $\beta_{1}$, ILK and $\alpha$-SMA mRNA expression were determined by real-time RT-PCR (Figure 3A). The expression levels of all of the examined molecules were higher in the diabetic glomeruli than in the control glomeruli. Benazepril treatment inhibited the expression of those molecules, but failed to rescue to the same level in the normal group. The western blot analysis showed a similar pattern of expression (Figure 3B and C). The control glomeruli showed a faint band of the ILK or $\alpha$-SMA protein, while the bands were stronger in the diabetic glomeruli. The bands in the benazepril treatment group 

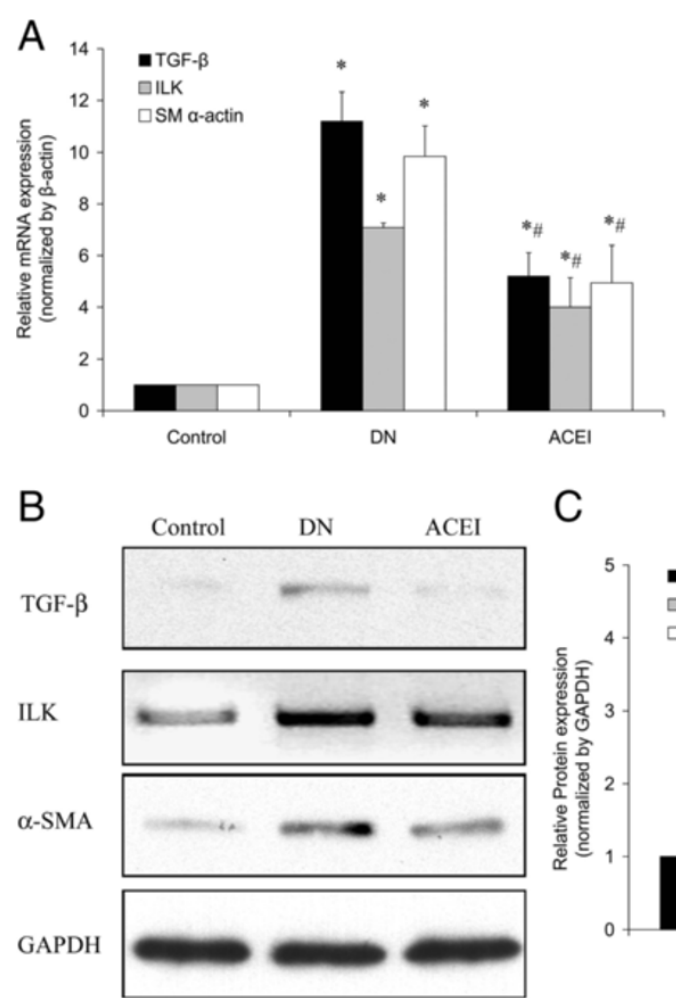

C

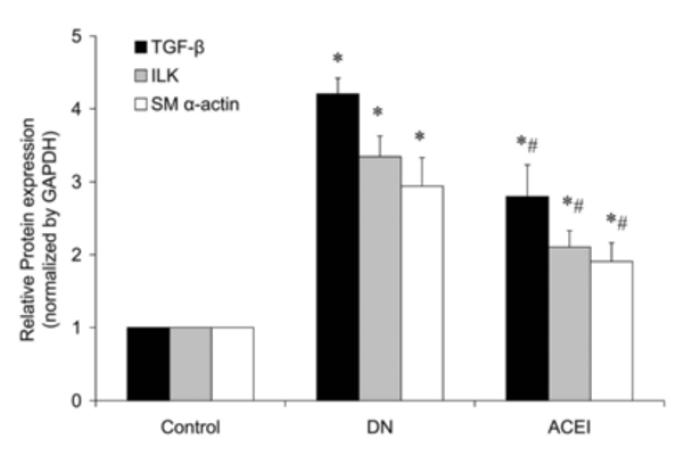

Figure 3 Benazepril affects TGF- $\beta_{1}$, ILK and $\boldsymbol{\alpha}$-SMA mRNA and protein expression in glomeruli. (A) TGF- $\beta_{1}$, ILK and a-SMA mRNA expression levels in the glomeruli of the NC, DN and ACEl group were determined by real-time RT-PCR. (B) ILK and a-SMA protein expression levels in the glomeruli of the different groups were determined by western blot. (C) Representative western blot analysis. Densitometric quantification of the corresponding bands is performed using Kodak 1D Image software. The values are the means \pm SE and are expressed relative to the control. $n=12$. ${ }^{*} P<0.05$ vs. control; $\#, P<0.05$ vs. DN.

were lower than in the DN group but higher than in the normal group (Figure 3B and C).

\section{Benazepril affects TGF- $\beta_{1}$, ILK and $\alpha$-SMA expression in cultured GMCs}

The RT-PCR results showed that HG levels induced ILK mRNA in a time-dependent manner compared with the NG. The ILK mRNA expression began to increase at $3 \mathrm{~h}$ and reached a peak at $48 \mathrm{~h}$ after treatment. The ILK mRNA expression began to decrease at $72 \mathrm{~h}$ but remained higher than the levels in the NG and HG at each time point (Figure 4A). Benazepril treatment did not completely attenuate the HG induced ILK mRNA expression at $48 \mathrm{~h}$ or $72 \mathrm{~h}$, and the levels remained higher than in the NG. There were no significant differences between the MG and NG groups. The expression of $\alpha$-SMA mRNA showed a similar pattern (Figure 4B). The western blotting analysis revealed that ILK and $\alpha$-SMA protein expression in the HG started to increase as early as $6 \mathrm{~h}$ (ILK, $P<$ $0.05)$ or $24 \mathrm{~h}(\alpha$-SMA, $P<0.05)$ and reached a peak at $48 \mathrm{~h}$ (Figure $4 \mathrm{C}-\mathrm{F}$ ). Benazepril treatment inhibited the HG induced ILK and $\alpha$-SMA protein expression in the ACEI group at $48 \mathrm{~h}$, but it remained higher than in the NG or MG (Figure $4 \mathrm{C}$-F). We found that the expression levels of $\alpha$-SMA mRNA and protein in the MG were increased as early as $6 \mathrm{~h}$ (mRNA, Figure 4B) or $24 \mathrm{~h}$ (Protein, Figure $4 \mathrm{E}$ and $\mathrm{F}$ ), which was higher than in the NG $(P<0.05)$, suggesting that high osmotic pressure might cause the increase in the $\alpha$-SMA expression [20].

To confirm the effect of benazepril on the TGF- $\beta_{1}$ mRNA expression and ILK and $\alpha$-SMA protein synthesis, RNA and protein were isolated from GMCs after being cultured in NG, MG, HG, or ACEI for $48 \mathrm{~h}$. The results showed that $\mathrm{HG}$ levels upregulated TGF- $\beta_{1}$ mRNA expression, and benazepril treatment effectively blocked the HG induced TGF- $\beta_{1}$ mRNA expression (Figure 5A). The western blot analysis showed that the HG levels increased the ILK and $\alpha$-SMA expression, parallel with TGF- $\beta_{1}$ mRNA expression in response to Benazepril, suggesting that the expression of those molecules was affected by HG induced TGF- $\beta_{1}$ expression (Figure 5B and C).

\section{Benazepril affects Ang II signalling pathways in GMCs}

High-glucose exposure can increase Ang II generation in cultured GMCs [21]. To elucidate the mechanism(s) of the effect of benazepril on GMC cellular processes, we assessed the effect of benazepril on Ang II signalling 


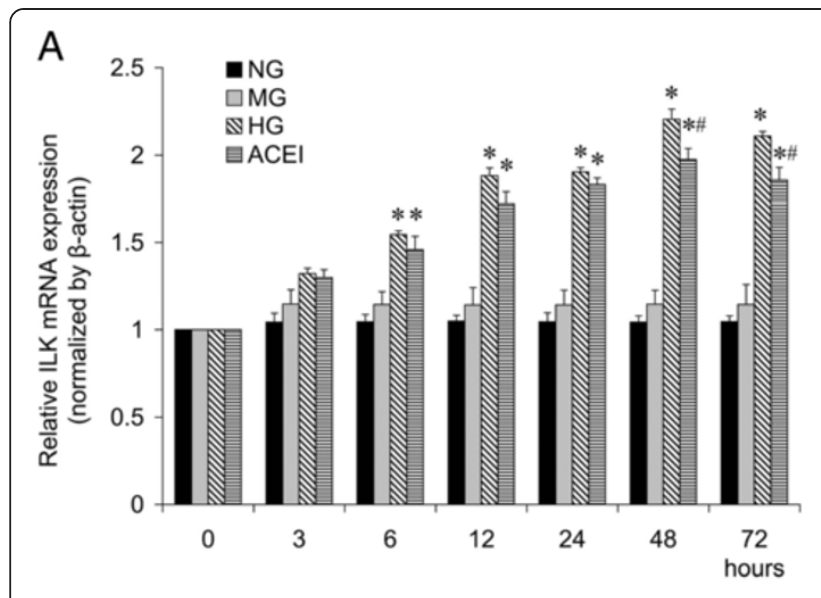

C ILK

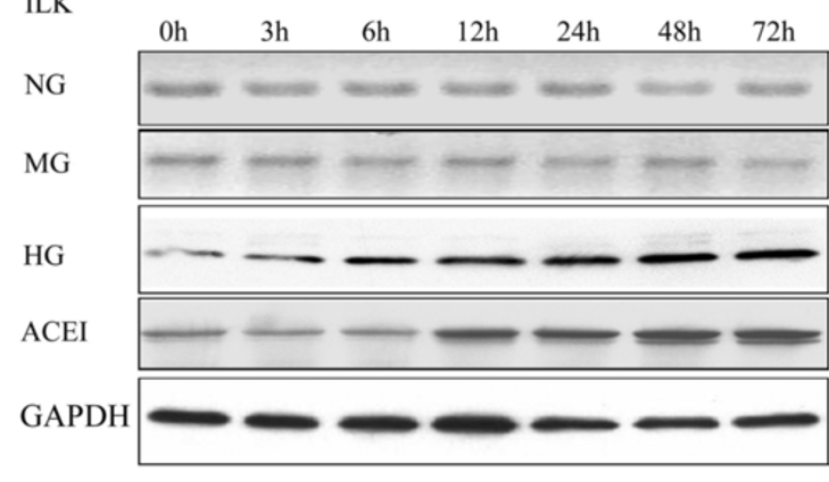

$\mathrm{E}$ $\alpha-$ SMA

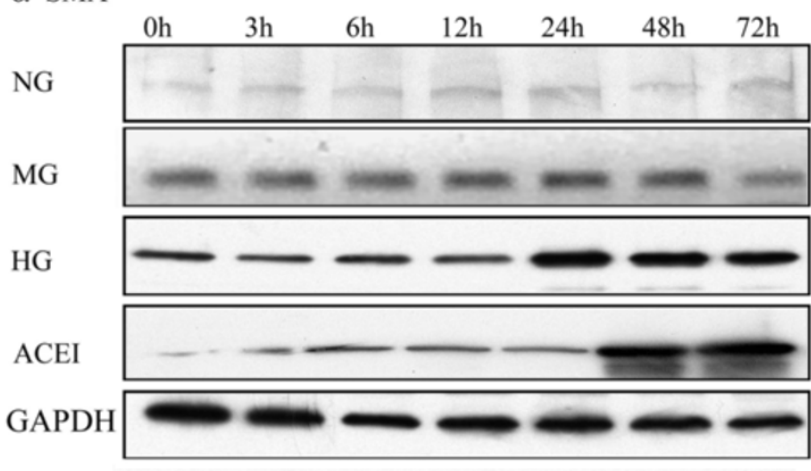

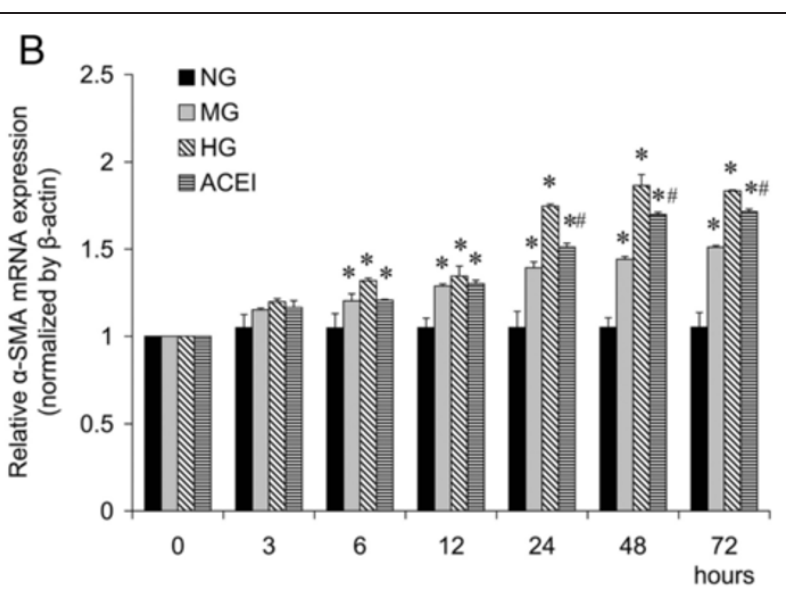

D
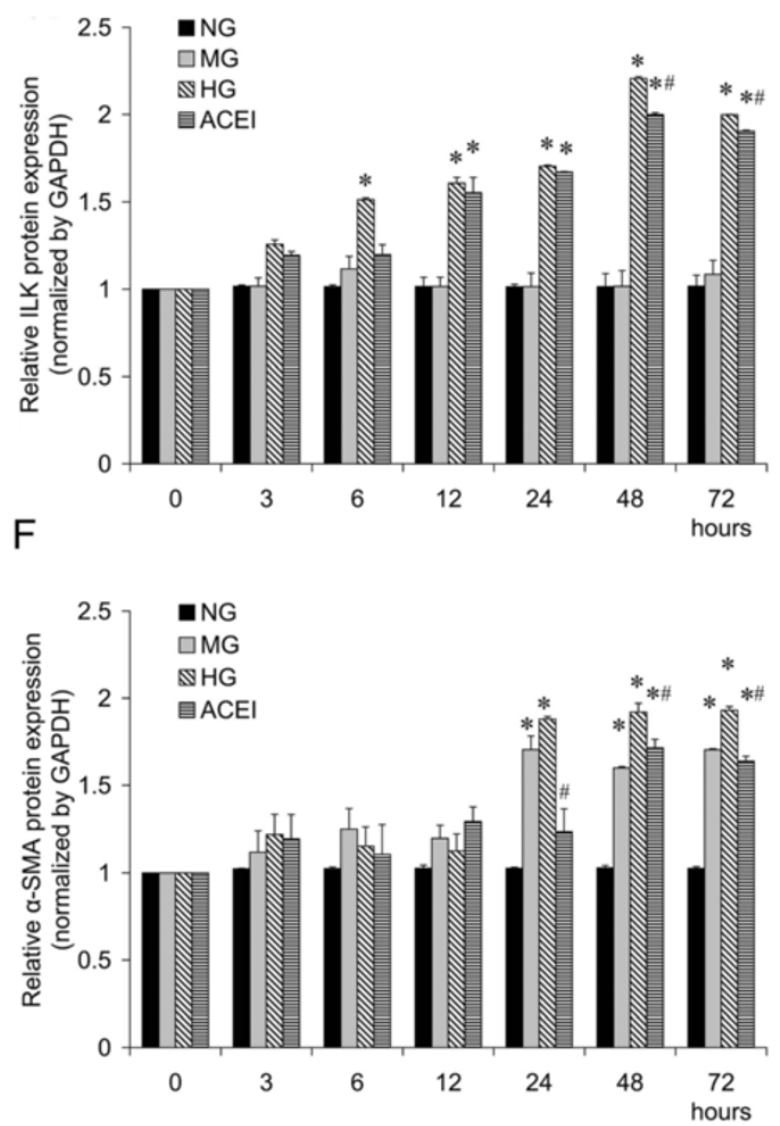

Figure 4 Benazepril affects the TGF- $\beta_{1}$, ILK and a-SMA expression in glomerular mesangial cell (GMC). Benazepril affects the expression of ILK (A) and a-SMA (B) mRNA in GMCs under HG conditions at different times, as determined by real-time RT-PCR. Benazepril affects the expression of ILK (C and $\mathbf{D}$ ) and a-SMA (E and $\mathbf{F}$ ) proteins in GMCs under HG conditions at different time points, as determined by western blot analysis. Densitometric quantification of the corresponding bands is performed using Kodak 1D Image software. The values are the means \pm SE and are expressed relative to the control. $n=6 .{ }^{*}, P<0.05$ vs. control; $\#, P<0.05$ vs. DN.

pathways. ERK activation supports GMC proliferation, while Akt phosphorylation mediates cell growth and TGF- $\beta$ gene transcription [22]. Western blotting showed that HG levels enhanced Ang II-induced ERK and Akt phosphorylation in GMCs (Figure 6A-C). Mannitol did not have a significant induction effect on the basal phosphorylation of ERK or Akt at the same time point. Benazepril significantly attenuated the HG induced ERK and 

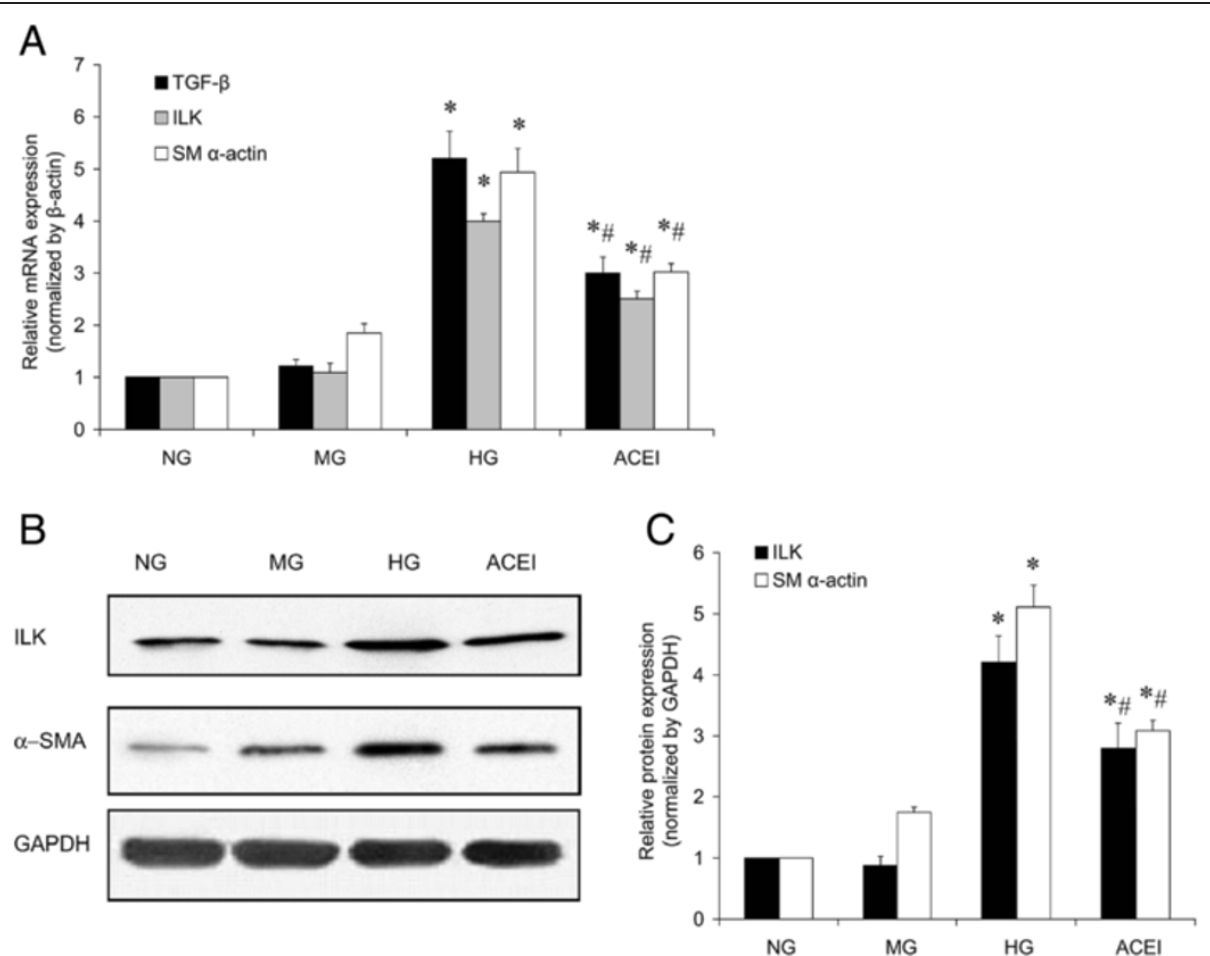

Figure 5 Benazepril treatment inhibited the high glucose induced TGF- $\beta_{1}$, ILK and $\boldsymbol{a}$-SMA expression at 48 h. (A) The TGF- $\beta_{1}$, ILK and a-SMA mRNA expression in NG, MG, HG and ACEI were determined by real-time RT-PCR at $48 \mathrm{~h}$. (B) Western blotting determined ILK and a-SMA protein expression in the $\mathrm{NG}, \mathrm{MG}, \mathrm{HG}$ and $\mathrm{ACEl}$ at $48 \mathrm{~h}$. (C) A representative western blot analysis. Densitometric quantification of the corresponding bands is performed using Kodak 1D Image software. Values are means \pm SE and are expressed relative to the control. $n=6$. *, $P<0.05$ vs control; $\#, P<0.05$ vs DN.

Akt phosphorylation, indicating that benazepril affected the GMC function through Ang II signalling in the GMCs (Figure 6A-C).

\section{Discussion}

DN is a common cause of end-stage kidney disease worldwide. The characteristic early abnormalities of diabetic kidneys are increased renal size and hyperfiltration. With the alteration of the glomerular filtration barrier, the glomerular structure collapses and leads to an increase in the albumin excretion rate followed by the development of GMC proliferation, ECM accumulation, and glomerular sclerosis. GMC proliferation is often considered an initial, adaptive response that eventually loses control and develops into a pathological process [23,24]. HG induced autocrine or paracrine variety growth factors, cytokines, chemokines and vasoactive agents, including TGF- $\beta_{1}$ and Ang II, have been implicated in the stimulation of ECM accumulation following structural changes of DN. TGF- $\beta_{1}$ expression was increased in experimental diabetic animals and diabetic patients. Anti-TGF- $\beta_{1}$ antibody or TGF- $\beta_{1}$ antisense oligonucleotides attenuated renal hypertrophy or HG induced GMC FN expression by inhibition of ECM gene expression. Ang II can induce TGF- $\beta_{1}$ expression in GMCs, suggesting that TGF- $\beta_{1}$ is the final common mediator of $\mathrm{DN}[10,25,26]$. ILK plays an important role in the interface between TGF- $\beta_{1}$, ECM, the actin-based cytoskeleton and the cellular phenotype in kidney diseases [27]. We determined that ILK expression increased in the renal tissue of DN rats or in HG treated GMCs, indicating that HG levels induced ILK expression at least in part through increasing TGF- $\beta_{1}$ autocrine secretion. Benazepril could attenuate the HG level induced TGF- $\beta_{1}$ and ILK expression in vivo or in vitro, suggesting that Ang II also affects TGF- $\beta_{1}$ and ILK expression.

GMCs that are activated by local injury impaired the activation of $\alpha$-SMA expression following GMC proliferation and basement membrane remodelling, which affects the glomerular filtration. Diabetes was shown to produce an increase in the expression of $\alpha$-SMA in the kidney glomeruli and to result in the accumulation of type IV collagen, resulting in renal fibrosis and nephropathy $[6,28]$. We observed that $\alpha$-SMA expression was significantly increased in the DN rat, and HG levels increased the $\alpha$ SMA mRNA and protein levels in GMCs with a timedependent pattern. The increased $\alpha$-SMA expression was an important step in the GMC phenotypic changes from the non-activated state to the proliferative, secretory activated state. Activated GMCs increased ECM production, increased inflammatory response, increased their own 


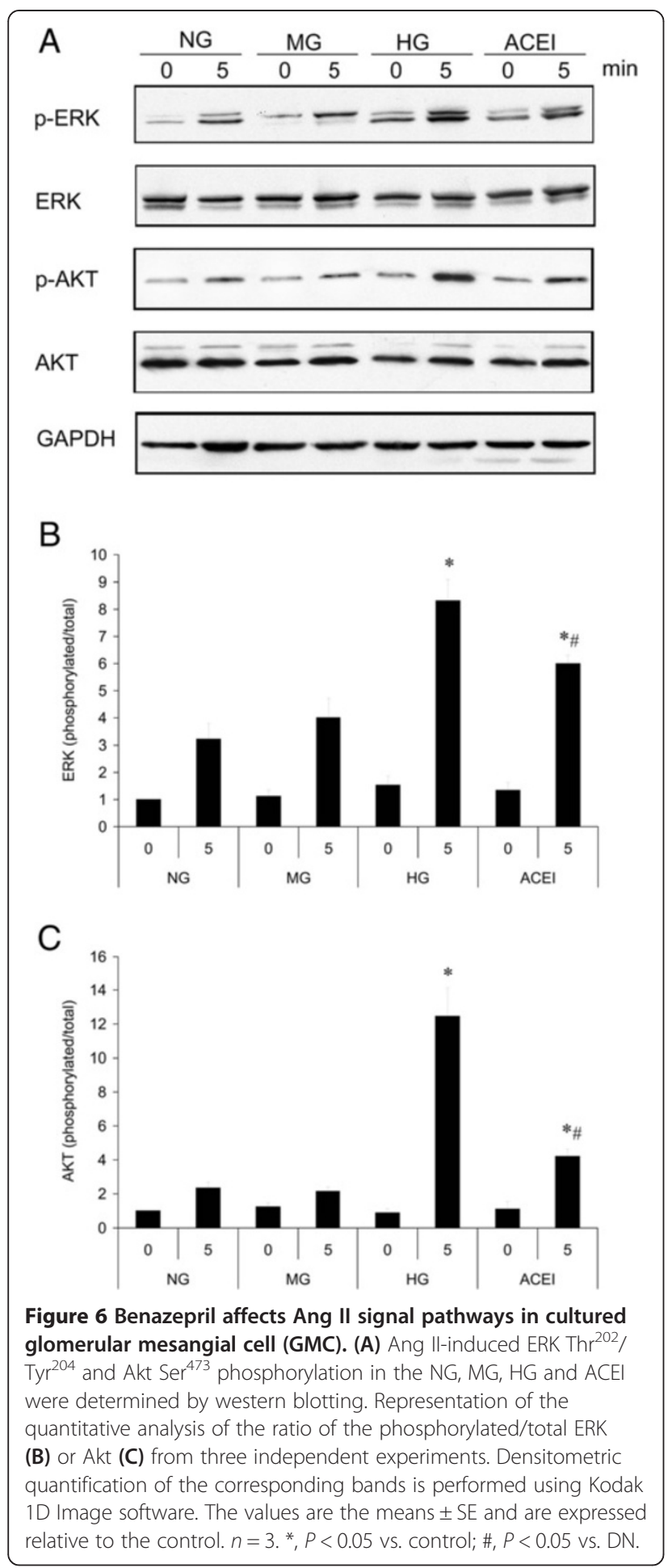

proliferation, and lead to glomerulosclerosis [29]. HG levels induced Ang II generation in cultured GMCs [21]. Ang II increases vascular resistance, reduces renal blood flow, and stimulates the production of ECM in the GMCs, which is one of the abnormalities in diabetic renal disease $[9,30]$. The ERK pathway was shown to be crucial in cell proliferation and differentiation, which is considered to be an important molecular mechanism in the development and progression of DN [31,32]. The Akt pathway is a critical regulator for a variety of cellular processes, including cell growth, cell motility, and TGF- $\beta_{1}$ gene transcription in GMCs [33,34]. We determined that HG levels enhanced Ang II-induced ERK and Akt phosphorylation in GMCs. Benazepril significantly attenuated the HG induced ERK and Akt phosphorylation. We found TGF- $\beta_{1}$ expression increased in the glomeruli of diabetic rats and in cultured GMCs under HG.

Benazepril, a kind of multifunction drug, primarily used in treatment of hypertension, congestive heart failure, and heart attacks, and also has beneficial effects in preventing renal and retinal complications of diabetes [35]. ACEI treatment showed lower in body weight, lower blood pressure, and a bit unexpectedly lower blood glucose levels than DN group (Table 1). ACE-I treated group was really specific to the inhibition of the renin-angiotensin-aldosterone system rather than secondary to i) lower body weight, thus less hyperfiltration and less glomerular hypertrophy, ii) less hyperglycemia and most importantly iii) lower blood pressure. All of these factors are well known driving factors behind the development of DN. For the therapeutic effect, ACEI group, the real control of DN group, showed the renoprotective function in the development of $\mathrm{DN}$, but still a diabetic group treated with e.g. a thiazide diuretic to lower blood pressure level to a similar degree as seen in the treatment group should be used in the future, which it helps a great deal to illustrate that our results remain consistent across the data in vivo and in vitro.

\section{Conclusions}

ERK and Akt play roles in the development and progression of $\mathrm{DN}$, and they might be potential therapeutic targets. The findings described here support the hypothesis that the high-glucose milieu of diabetes increases TGF$\beta_{1}$ secretion, which increases the synthesis of ILK and $\alpha$ SMA that are involved in the progression of DN. This might be an important mechanism of the benazepril renoprotective function in the pathogenesis of $\mathrm{DN}$.

\section{Additional file}

Additional file 1: The ARRIVE Guidelines Checklist.

\section{Abbreviations}

DN: Diabetic nephropathy; ACEl: Angiotensin converting enzyme inhibitor; TGF- $\beta_{1}$ : Transforming growth factor- $\beta_{1}$; ILK: Integrin-linked kinase; GMC: Glomerular mesangial cell; FN: Fibronectin; a-SMA: Smooth muscle a-actin; Ang II: Angiotensin II; ECM: Extracellular matrix.

\section{Competing interests}

The authors declare that they have no competing interests. 


\section{Authors' contributions}

HLN performed the experiments and data analysis, participated in the design of the dtudy and drafted the manuscript. LN participated in its design and coordination and helped to draft the manuscript. MDL contributed to the design of the sutdy and helped wth perfoming eht experiments and drafting the manuscripe. YQC and TZ provided intellectual imput to the study and helped with the revision of the manuscript. YL conceived of and designed the study and supervided the work. All authors read and approved the final manuscript.

\section{Acknowledgements}

This study was supported, in part, by the grants obtained from Hebei Natural Science Foundation (No.C20080000940) and Technology Commission Foundation (No.072761229).

\section{Author details}

'Department of Nephrology, The Third Hospital of Hebei Medical University, Shijiazhuang 050051, China. 'Key Laboratory of Kidney Diseases of Hebei Province, Shijiazhuang 050071, China. ${ }^{3}$ Cardiovascular Molecular Imaging Laboratory, Section of Cardiovascular Medicine, Yale University, New Haven, Connecticut 06511, USA. "VA Connecticut Healthcare System, West Haven, Connecticut 06519, USA.

Received: 24 March 2014 Accepted: 13 August 2014

Published: 20 August 2014

\section{References}

1. Remuzzi G, Schieppati A, Ruggenenti P: Clinical practice. Nephropathy in patients with type 2 diabetes. N Engl J Med 2002, 346(15):1145-1151.

2. Sego S: Pathophysiology of diabetic nephropathy. Nephrol Nurs J 2007, 34(6):631-633.

3. Kanwar YS, Wada J, Sun L, Xie P, Wallner El, Chen S, Chugh S, Danesh FR: Diabetic nephropathy: mechanisms of renal disease progression. Exp Biol Med (Maywood) 2008, 233(1):4-11.

4. Kim SM, Kim N, Lee S, Kim do K, Lee YM, Ahn SH, Song JH, Choi BK, Wu C, Jung KY: TGF-beta1-induced PINCH-1-ILK-alpha-parvin complex formation regulates mesangial cell proliferation and hypertrophy. Exp Mol Med 2007, 39(4):514-523.

5. Guo L, Sanders PW, Woods A, Wu C: The distribution and regulation of integrin-linked kinase in normal and diabetic kidneys. Am J Pathol 2001, 159(5):1735-1742.

6. Carey AV, Carey RM, Gomez RA: Expression of alpha-smooth muscle actin in the developing kidney vasculature. Hypertension 1992, 19(2 Suppl):I1168-175.

7. Hu P, Qin YH, Pei J, Lei FY, Hu B, Lu L: Beneficial effect of all-trans retinoic acid (ATRA) on glomerulosclerosis rats via the down-regulation of the expression of alpha-smooth muscle actin: a comparative study between ATRA and benazepril. Exp Mol Pathol 2010, 89(1):51-57.

8. Jacobson HR: Chronic renal failure: pathophysiology. Lancet 1991, 338(8764):419-423

9. Leehey DJ, Singh AK, Alavi N, Singh R: Role of angiotensin II in diabetic nephropathy. Kidney Int Suppl 2000, 77:S93-98.

10. Border WA, Noble NA: Evidence that TGF-beta should be a therapeutic target in diabetic nephropathy. Kidney Int 1998, 54(4):1390-1391.

11. Parving HH: Diabetic nephropathy: prevention and treatment. Kidney Int 2001, 60(5):2041-2055.

12. Jacobsen P, Rossing K, Parving HH: Single versus dual blockade of the renin-angiotensin system (angiotensin-converting enzyme inhibitors and/or angiotensin II receptor blockers) in diabetic nephropathy. Curr Opin Nephrol Hypertens 2004, 13(3):319-324.

13. Maschio G, Alberti D, Janin G, Locatelli F, Mann JF, Motolese M, Ponticelli C, Ritz E, Zucchelli P: Effect of the angiotensin-converting-enzyme inhibitor benazepril on the progression of chronic renal insufficiency. The Angiotensin-Converting-Enzyme Inhibition in Progressive Renal Insufficiency Study Group. N Engl J Med 1996, 334(15):939-945.

14. Satoh M, Fujimoto S, Haruna Y, Arakawa S, Horike H, Komai N, Sasaki T, Tsujioka K, Makino H, Kashihara N: NAD(P)H oxidase and uncoupled nitric oxide synthase are major sources of glomerular superoxide in rats with experimental diabetic nephropathy. Am J Physiol Renal Physiol 2005, 288(6):F1144-1152
15. Parasuraman $S$, Raveendran R: Measurement of invasive blood pressure in rats. J Pharmacol Pharmacother 2012, 3(2):172-177.

16. Watson AM, Gray SP, Jiaze L, Soro-Paavonen A, Wong B, Cooper ME, Bierhaus A, Pickering R, Tikellis C, Tsorotes D, Thomas MC, Jandeleit-Dahm KA: Alagebrium reduces glomerular fibrogenesis and inflammation beyond preventing RAGE activation in diabetic apolipoprotein $\mathrm{E}$ knockout mice. Diabetes 2012, 61(8):2105-2113.

17. Okuda S, Languino LR, Ruoslahti E, Border WA: Elevated expression of transforming growth factor-beta and proteoglycan production in experimental glomerulonephritis. Possible role in expansion of the mesangial extracellular matrix. J Clin Invest 1990, 86(2):453-462.

18. Huang $H$, Li Y, Liu M, Shi Y, Zhang T: Gremlin induces cell proliferation and extra cellular matrix accumulation in mouse mesangial cells exposed to high glucose via the ERK1/2 pathway. BMC Nephrol 2013, 14(33):1471-2369.

19. Border WA, Ruoslahti E: Transforming growth factor-beta in disease: the dark side of tissue repair. J Clin Invest 1992, 90(1):1-7.

20. Sheetz MJ, King GL: Molecular understanding of hyperglycemia's adverse effects for diabetic complications. Jama 2002, 288(20):2579-2588.

21. Vidotti DB, Casarini DE, Cristovam PC, Leite CA, Schor N, Boim MA: High glucose concentration stimulates intracellular renin activity and angiotensin II generation in rat mesangial cells. Am J Physiol Renal Physiol 2004, 286(6):F1039-1045

22. Mondorf UF, Geiger H, Herrero M, Zeuzem S, Piiper A: Involvement of the platelet-derived growth factor receptor in angiotensin II-induced activation of extracellular regulated kinases 1 and 2 in human mesangial cells. FEBS Lett 2000 472(1):129-132

23. Wesson LG: Physical factors and glomerulosclerosis. Cause or coincidence? Nephron 1998, 78(2):125-130.

24. Lopez-Novoa JM, Rodriguez-Pena AB, Ortiz A, Martinez-Salgado C, Lopez Hernandez FJ: Etiopathology of chronic tubular, glomerular and renovascular nephropathies: clinical implications. J Trans/ Med 2011, 9:13.

25. Kolm V, Sauer U, Olgemoller B, Schleicher ED: High glucose-induced TGF-beta 1 regulates mesangial production of heparan sulfate proteoglycan. $\mathrm{Am} J$ Physiol 1996, 270(5 Pt 2):F812-821.

26. Oh JH, Ha H, Yu MR, Lee HB: Sequential effects of high glucose on mesangial cell transforming growth factor-beta 1 and fibronectin synthesis. Kidney Int 1998, 54(6):1872-1878.

27. Blattner SM, Kretzler M: Integrin-linked kinase in renal disease: connecting cell-matrix interaction to the cytoskeleton. Curr Opin Nephrol Hypertens 2005, 14(4):404-410

28. Sanai T, Sobka T, Johnson T, el-Essawy M, Muchaneta-Kubara EC, Ben Gharbia O, el Oldroyd S, Nahas AM: Expression of cytoskeletal proteins during the course of experimental diabetic nephropathy. Diabetologia 2000, 43(1):91-100.

29. Xin C, Ren S, Eberhardt W, Pfeilschifter J, Huwiler A: The immunomodulator FTY720 and its phosphorylated derivative activate the Smad signalling cascade and upregulate connective tissue growth factor and collagen type IV expression in renal mesangial cells. Br J Pharmacol 2006, 147(2):164-174.

30. Burns KD: Angiotensin II and its receptors in the diabetic kidney. Am J Kidney Dis 2000, 36(3):449-467.

31. Awazu M, Ishikura K, Hida M, Hoshiya M: Mechanisms of mitogenactivated protein kinase activation in experimental diabetes. J Am Soc Nephrol 1999, 10(4):738-745.

32. Haneda M, Araki S, Togawa M, Sugimoto T, Isono M, Kikkawa R: Mitogenactivated protein kinase cascade is activated in glomeruli of diabetic rats and glomerular mesangial cells cultured under high glucose conditions. Diabetes 1997, 46(5):847-853.

33. Delcommenne M, Tan C, Gray V, Rue L, Woodgett J, Dedhar S: Phosphoinositide-3-OH kinase-dependent regulation of glycogen synthase kinase 3 and protein kinase B/AKT by the integrin-linked kinase. Proc Natl Acad Sci U S A 1998, 95(19):11211-11216.

34. Remy I, Montmarquette A, Michnick SW: PKB/Akt modulates TGF-beta signalling through a direct interaction with Smad3. Nat Cell Biol 2004, 6(4):358-365.

35. Hou FF, Zhang X, Zhang GH, Xie D, Chen PY, Zhang WR, Jiang JP, Liang M, Wang GB, Liu ZR, Geng RW: Efficacy and safety of benazepril for advanced chronic renal insufficiency. N Engl J Med 2006, 354(2):131-140.

doi:10.1186/1471-2369-15-135

Cite this article as: Niu et al:: Benazepril affects integrin-linked kinase and smooth muscle a-actin expression in diabetic rat glomerulus and cultured mesangial cells. BMC Nephrology 2014 15:135. 